OPEN ACCESS

Edited by:

James Shorter,

University of Pennsylvania, USA

Reviewed by:

Andre Hoelz,

California Institute of Technology, USA

Kürşad Turgay,

Leibniz University of Hanover,

Germany

*Correspondence:

Christian Schlieker

christian.schlieker@yale.edu

Specialty section:

This article was submitted to

Protein Folding, Misfolding and

Degradation,

a section of the journal

Frontiers in Molecular Biosciences

Received: 25 March 2017 Accepted: 25 April 2017 Published: 11 May 2017

Citation:

Chase AR, Laudermilch E and Schlieker C (2017) Torsin ATPases: Harnessing Dynamic Instability for Function. Front. Mol. Biosci. 4:29. doi: 10.3389/fmolb.2017.00029

\section{Torsin ATPases: Harnessing Dynamic Instability for Function}

\author{
Anna R. Chase ${ }^{1}$, Ethan Laudermilch ${ }^{1}$ and Christian Schlieker ${ }^{1,2 *}$ \\ ${ }^{1}$ Department of Molecular Biophysics and Biochemistry, Yale University, New Haven, CT, USA, ${ }^{2}$ Department of Cell Biology, \\ Yale School of Medicine, New Haven, CT, USA
}

Torsins are essential, disease-relevant AAA+ (ATPases associated with various cellular activities) proteins residing in the endoplasmic reticulum and perinuclear space, where they are implicated in a variety of cellular functions. Recently, new structural and functional details about Torsins have emerged that will have a profound influence on unraveling the precise mechanistic details of their yet-unknown mode of action in the cell. While Torsins are phylogenetically related to $\mathrm{Clp} / \mathrm{HSP} 100$ proteins, they exhibit comparatively weak ATPase activities, which are tightly controlled by virtue of an active site complementation through accessory cofactors. This control mechanism is offset by a TorsinA mutation implicated in the severe movement disorder DYT1 dystonia, suggesting a critical role for the functional Torsin-cofactor interplay in vivo. Notably, TorsinA lacks aromatic pore loops that are both conserved and critical for the processive unfolding activity of Clp/HSP100 proteins. Based on these distinctive yet defining features, we discuss how the apparent dynamic nature of the Torsin-cofactor system can inform emerging models and hypotheses for Torsin complex formation and function. Specifically, we propose that the dynamic assembly and disassembly of the Torsin/cofactor system is a critical property that is required for Torsins' functional roles in nuclear trafficking and nuclear pore complex assembly or homeostasis that merit further exploration. Insights obtained from these future studies will be a valuable addition to our understanding of disease etiology of DYT1 dystonia.

Keywords: AAA+ proteins, TorsinA, dystonic disorders, nuclear membrane, nuclear pore complex, DYT1 dystonia, protein quality control, ubiquitin

\section{INTRODUCTION}

Torsin ATPases are essential and broadly conserved AAA+ proteins whose discovery was tied to the characterization of the TorsinA DYT1 mutation found in patients with early-onset torsion dystonia, a highly debilitating hereditary movement disorder (Ozelius et al., 1997). Torsins have recently garnered increasing interest in conjunction with pivotal discoveries about their structure and molecular mechanism of activation, as well as compelling insights into their cellular functions. As the sole AAA+ ATPase found in the endoplasmic reticulum (ER) and nuclear envelope (NE), Torsins were implicated in equally broad and critical functions including lipid synthesis (Grillet et al., 2016), regulation of membrane morphology (Rose et al., 2014), and protein quality control (Chen et al., 2010; Nery et al., 2011) as well as the ER redox sensing (Zhu et al., 2008, 2010; Nery et al., 2011; Zhao et al., 2016).

In addition to these roles in the ER, Torsins fulfill distinct functions at the NE. TorsinA and its cofactor LAP1 are essential for proper assembly of fibroblast nuclear envelope-anchored 
transmembrane actin-associated nuclear (TAN) lines (Luxton et al., 2011), which are comprised of arrays of linker of nucleoskeleton and cytoskeleton (LINC) complexes associated with retrograde flowing actin. Torsin A modulates the rearward motion of nuclei during centrosome positioning and is implicated in maintaining cell polarity in migrating cells (Saunders et al., 2017). A second intriguing role for Torsins at the nuclear periphery is their involvement in modulating nuclear envelope architecture. Deletions of Torsins in human, mouse, worm, and fly cells lead to the formation of omega-shaped "bleb" compartments within the nuclear envelope (Goodchild et al., 2005; Jokhi et al., 2013; Liang et al., 2014; VanGompel et al., 2015; Laudermilch et al., 2016; Tanabe et al., 2016). These perinuclear blebs have been shown to harbor ubiquitinated proteins (Liang et al., 2014; Laudermilch et al., 2016) as well as nuclear pore complex components (Laudermilch et al., 2016). Thus, a picture is emerging in which Torsins accomplish a variety of tasks both at the NE and the ER, and that at least some of these functions are most critical during early developmental stages in neurons (Tanabe et al., 2016). In addition to these functional insights in the cellular context, the recently solved crystal structures of wild-type and DYT1 dystonia mutant Torsin in complex with its cofactor LULL1 confirmed functionally significant structural features that were previously unappreciated (Demircioglu et al., 2016). Several reviews have summarized the current state of the Torsin field (Rose et al., 2015; Laudermilch and Schlieker, 2016; Cascalho et al., 2017); thus, the purpose of the forgoing is to spotlight current hypotheses surrounding the Torsins' roles at the inner nuclear membrane and their dynamic assembly into an active, functional complex.

\section{STRUCTURAL INSIGHTS INTO TORSIN COMPLEXES}

Though homology to other AAA+ proteins suggested that Torsins were capable of ATP hydrolysis-driven mechanical work from the very beginning, the question of whether they were active ATPases or degenerate AAA + scaffolds was unresolved until Torsins were functionally reconstituted in vitro (Zhao et al., 2013). Torsin A, -B, and -3A have ATPase activity in the presence of ATP and the luminal domain of the ER-resident protein LULL1 while Torsin $\mathrm{A}$ and $-\mathrm{B}$ alone are activated by the luminal domain of LAP1, which resides in the NE (Foisner and Gerace, 1993; Goodchild and Dauer, 2005; Zhao et al., 2013). The DYT1 dystonia mutant of TorsinA is refractory to the activation by these cofactors, thus presenting one line of evidence for a loss-of-function mechanism in early-onset torsion dystonia (Zhao et al., 2013). These cofactors have degenerate $\mathrm{AAA}+$ scaffolds lacking the motifs needed for ATP binding, and they activate Torsin ATPase activity by complementing the Torsin active site with an arginine finger residue that is absent in Torsins (Brown et al., 2014; Sosa et al., 2014) (Figures 1A-C).

The structure of the TorsinA-LULL1 heterodimer unambiguously confirmed the critical role of a catalytic arginine (Demircioglu et al., 2016). This arginine is positioned to stabilize the negative charge of the transition state, thus lowering the free energy of the nucleotide hydrolysis reaction (Scheffzek et al., 1998). As suggested by biochemical studies (Brown et al., 2014; Rose et al., 2014) the TorsinA-LULL1 crystal structure confirmed the critical role of Torsin's C-terminal helix region for forming interactions with LULL1 (Demircioglu et al., 2016) (Figure 1C). It is now apparent that the deletion of E303 in the DYT1 dystonia mutant TorsinA perturbs a critical helix at the cofactor interface (Demircioglu et al., 2016), providing an atomic-level rationale for the observation of reduced cross-linking of the conserved C-terminal Torsin A aromatic residues with the cofactor in the Torsin A disease variant (Brown et al., 2014), and the resulting failure to trigger ATP hydrolysis (Zhao et al., 2013) (for additional details on disease implications, see Rose et al., 2015; Cascalho et al., 2017).

The complementation mechanism for ATPase activation and the presence of a degenerated $\mathrm{AAA}+$ fold is unusual but not unprecedented. The bacterial clamp loader has an inactive $\delta^{\prime}$ subunit that activates the adjacent $\gamma$ ATP-binding AAA+ subunit (Hedglin et al., 2013; Kelch, 2016). Torsins and their cofactors stand out for the fact that they have different modes of staying anchored in their cellular environment: Torsin A and -B have an N-terminal signal sequence followed by a hydrophobic domain while Torsin $2 \mathrm{~A}$ and $-3 \mathrm{~A}$ do not have a hydrophobic domain, and LULL1 and LAP1 are type-II transmembrane proteins. LULL1 is localized throughout the ER (Goodchild and Dauer, 2005), while the nuclear domain of LAP1 binds to the nuclear lamina and therefore resides in the inner nuclear membrane (Foisner and Gerace, 1993). From an evolutionary standpoint, the added complexity of such a distinctive multicomponent ATPase system likely evolved out of the need to create more diverse roles at precise cellular loci, especially in higher organisms. Dependence on the cofactors for at least some of their functions likely allows cells to leverage the common Torsin scaffold to perform more varied functions in targeted locations and potentially relay signals from or to the nucleus and cytoplasm as well.

Though the stoichiometry of the Torsin/cofactor complex under equilibrium conditions remain to be established, recent data point to a dynamic assembly. Three distinct models exist: (a) an alternating, symmetric Torsin/cofactor ring assembly; (b) homo-oligomeric Torsin rings; and (c) a Torsin/cofactor dimer (Figure 1D). Though low-resolution structural (Sosa et al., 2014) data and crosslinking data (Brown et al., 2014) are consistent with the formation of an alternating assembly into a closed ring structure, the major limitation of several approaches aimed at a determination of the (hetero)oligomeric state is that they were mostly carried out with hydrolysis-deficient "trap" variants of TorsinA. These variants are refractory to cofactor-induced hydrolysis (Zhao et al., 2013) and bind the cofactor tightly (Naismith et al., 2009; Zhu et al., 2010; Zhao et al., 2013), a situation that is certainly not representative of the dynamic equilibrium in a cell. The rationale for the second model with Torsin-Torsin homo-oligomers is based on data showing that Torsin assembles into hexameric structures on its own in blue native PAGE experiments, and that ATP is often required to allow 
A

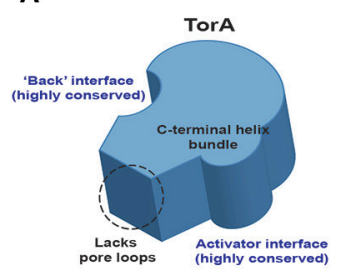

D

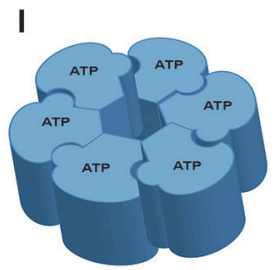

B

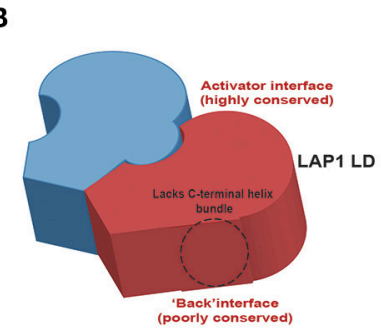

II

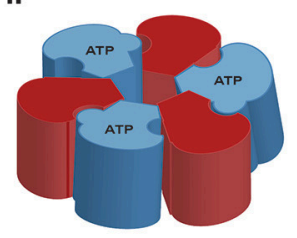

c

III

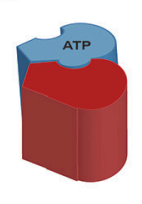

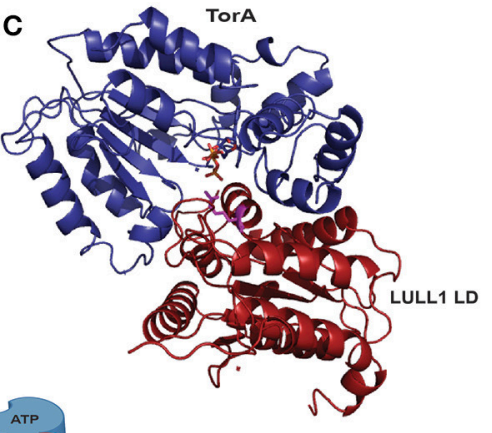

E

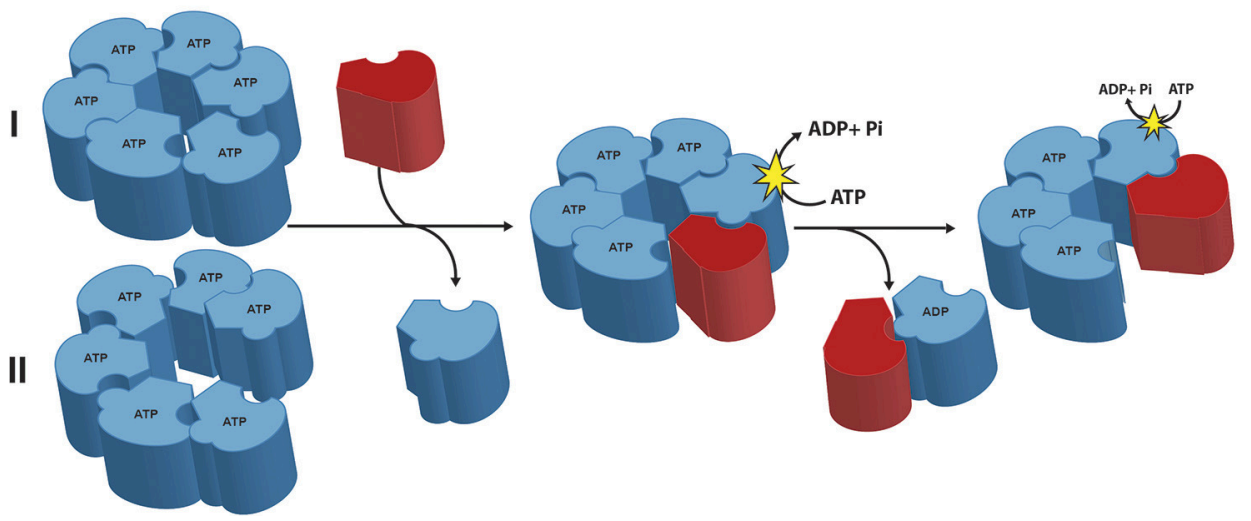

FIGURE 1 | Structural features of TorsinA and its dynamic complexes with cofactors (A). TorsinA (blue) exhibits high levels of conservation both on the activator and "back" interface. Torsins have a C-terminal helix bundle that serves to form intra-protomer contacts in related AAA+ proteins but lack the aromatic pore loops that usually serve to thread substrates through the central pore. The membrane-associated $\mathrm{N}$-terminal hydrophobic domain was omitted for clarity. (B) The cofactor LAP1 (maroon) luminal domain, which adopts a AAA+ fold, lacks the critical four-helix bundle and exhibits a low level of conservation on its "back" interface opposite the more conserved activator binding face. (C) Cartoon representation of the TorsinA-LULL1 crystal structure (PDB code 5J1S; the nanobody used for crystallization was omitted for clarity). Note that the luminal domains of LAP1 and LULL1 are 60\% identical. The cofactor/Torsin complex features a tightly apposed interface in the presence of ATP (orange), with the cofactor supplying a catalytic arginine finger (magenta) that reaches into the nucleotide binding site of Torsin to activate its ATPase activity. (D) Three different models exist for the active assembly of Torsins: (I) a homo-oligomeric (likely hexameric) ring; (II) a trimer of heterodimers; (III) a Torsin-LAP1 heterodimer. (E) Predicted model of active Torsin complex formation with its cofactors. Torsin forms homo-oligomeric complexes in the presence of nucleotide that could adopt either a planar (I) or a stacked spiral (II) conformation. Cofactor binding to the Torsin active site destabilizes the Torsin ring. Torsin-Torsin rings are eventually dismantled because the cofactors lack the necessary four-helix bundle and conserved residues to form stable closed ring structures. The Torsin-cofactor complex is also transient and dynamic: ATP hydrolysis generates ADP-bound Torsin, destabilizing both the Torsin-Torsin and the Torsin-cofactor interaction. Note that the transmembrane domain of LAP1 was omitted for clarity.

oligomerization in AAA + ATPases (Hanson and Whiteheart, 2005; Vander Heyden et al., 2009; Jungwirth et al., 2010).

Given that previous studies of Torsins were conducted primarily with "trap" variants that resulted in more static models, we propose a more dynamic model. This model is most strongly supported by the following evidence: only Torsins, but not LAP1 and LULL1, possess the C-terminal helix bundle that is essential for intra-protomer ring-forming contacts (Figures 1A-C) among the Clp/Hsp100 AAA+ proteins (Mogk et al., 2003); the high level of conservation observed in Torsin residues on the "back" interface opposite the cofactor binding face (Figures 1A,B) (Demircioglu et al., 2016) suggesting that these residues participate in homotypic Torsin intra-protomer contacts; and the observation of higher-order Torsin oligomers (cf. Figure 1D) via blue native PAGE (Vander Heyden et al., 2009; Jungwirth et al., 2010; Goodchild et al., 2015). Given the cofactors' lack of a four-helix bundle and the low level of "back" interface conservation on either cofactor (Demircioglu et al., 2016), and the fact that Torsin oligomerization itself is ATPdependent, it is conceivable that activation of ATP hydrolysis by the bound cofactors would effectively disrupt homotypic intra-ring contacts, as proposed previously (Rose et al., 2015; Demircioglu et al., 2016).

One important point of discussion in the context of this model is how the cofactor luminal domains, which would effectively compete with other Torsin subunits in the ring for a nearly 
identical interface would manage to initially pervade the ring, gaining access to an ATP-bound Torsin subunit. One possibility (Figure 1E-II) is that Torsin oligomers adopt a split lock washer or spiral conformation, similar to NSF (Zhao et al., 2015), in which parts of the nucleotide binding face of Torsin would be rendered accessible to the cofactor. The flexibility of the unstructured region after the hydrophobic domain but before the $\mathrm{AAA}+$ domain (residues 44-57) could impart additional degrees of translational freedom (a $\sim 49 \AA$ radius of flexibility, based on $\mathrm{C} \alpha-\mathrm{C} \alpha$ distance) to Torsin subunits, thus also allowing the membrane-anchored cofactors to access the nucleotide binding site, which is about $30 \AA$ from the membrane-anchored Nterminus. Considering that ATP binding is broadly required for oligomerization in AAA+ ATPases, hydrolysis and transition to the ADP-bound state would shift the equilibrium to free Torsin and cofactor subunits (Figure 1E I-II). Adding to the complexity of the system is the fact that LULL1 has been shown to form higher-order structures (Goodchild et al., 2015), thus creating an equilibrium reaction between Torsin-engaged, free, and homo-oligomeric or otherwise engaged cofactors. Furthermore, it is possible that the cofactors are themselves regulated by an additional layer of control: for example via posttranslational modifications, through dynamic interactions with other proteins on either side of the membrane, or even within the lipid bilayer. In either case, the known properties of the Torsin-cofactor complex are not consistent with a static assembly.

Unlike the Clp/Hsp100 proteins which Torsins are most phylogenetically similar to, the Torsin structure (Demircioglu et al., 2016) further established that Torsins lack the central hydrophobic pore loops that are used to drive substrate translocation through the central channel of other related hexameric AAA+ proteins (Olivares et al., 2016). Combined with the extremely slow ATPase activity (0.006 nucleotides/s), relative to its $\mathrm{AAA}+$ counterparts which can hydrolyze $>1.3$ nucleotides/s (Martin et al., 2008), these observations render it improbable that Torsin acts in a processive manner to translocate substrates through the inner cavity of the Torsin ring (Zhao et al., 2013; Rose et al., 2015). Instead, Torsins likely interact with substrates with a more transient mechanism such as that of a holder chaperone that quickly binds and releases its substrates, either by lateral diffusion into the axial pore or by binding substrates at the periphery of its assembly. Determining the three dimensional structure of higher-order Torsin assemblies using e.g., cryo-electron microscopy might provide important insights in the future. Though characterizing the precise mechanisms of how ATP hydrolysis translates to work exerted on substrates remains challenging even for wellcharacterized $\mathrm{AAA}+$ proteins, recent studies on NSF, the yeast chaperone Hsp104, and mitochondrial Pex1/Pex6 by cryo-EM have revealed that progression through multiple asymmetric states in stacked spirals, open lock-washers, or more planar assemblies are key drivers for performing work during successive ATP hydrolysis events (Blok et al., 2015; Zhao et al., 2015; Yokom et al., 2016). Given the Torsins assembly's dynamic nature, predicted non-processive action, and similarity to clamp loaders, it is probable that the presence of asymmetric states will also play a role in its activation mechanism and should be accounted for in data analysis and interpretation. Asymmetric hydrolysis events could, for example, couple various asymmetric states to the insertion of the Torsins' own hydrophobic domains or interaction with the transmembrane cofactors, which could in turn modulate membrane curvature and remodeling or substrate interactions. It will be important to examine these states both in the presence and absence of cofactors and, once they have been identified, the Torsin substrates that have eluded the field thus far.

How can we begin to form a mechanistic explanation for the Torsins' exquisite spatiotemporal control during phases of neuronal development while also accounting for their redundancy (Laudermilch et al., 2016; Tanabe et al., 2016)? One likely scenario, is the formation of an anti-parallel gradient by the cofactors LULL1 in the ER and LAP1 at the nuclear envelope that dictate when and where Torsins are activated by cofactors to perform their function (Rose et al., 2015). LULL1 could activate Torsin's chaperone function in the ER, perhaps in response to a flux in redox potential or cofactor density in this compartment. The membrane association of TorsinA is controlled by cleavage of a scissile bond that removes the $\mathrm{N}$ terminal hydrophobic domain during $B$ cell differentiation (Zhao et al., 2016), suggesting an additional layer of control that could modulate substrate specificity, for example from membraneassociated to soluble ER-luminal species, during ER expansion. TorsinA species with a mass identical to this cleavage product have been observed in organ homogenates (Goodchild et al., 2005; Jungwirth et al., 2010).

\section{A NOVEL ROLE FOR TORSINS IN NUCLEAR PORE BIOGENESIS OR HOMEOSTASIS}

The hallmark phenotype seen upon Torsin manipulation or deletion is the "blebbing" or herniation of the inner nuclear membrane into the perinuclear space (Figure 2A; Goodchild et al., 2005; Jokhi et al., 2013; Liang et al., 2014; Pappas et al., 2015; VanGompel et al., 2015; Laudermilch et al., 2016; Tanabe et al., 2016). This phenotype has been observed in neural tissues of knockout mouse models of TorsinA (Goodchild and Dauer, 2005) and in HeLa cells with combined knockouts of multiple Torsins (Laudermilch and Schlieker, 2016). Similar herniations have also been observed after manipulation of the respective Torsin variants in Drosophila melanogaster and Caenorhabditis elegans (Jokhi et al., 2013; VanGompel et al., 2015), suggesting that Torsin function at the nuclear envelope is conserved.

One formidable challenge to deciphering Torsin function has been the remarkable redundancy between the four Torsin proteins encoded in mammalian genomes. In TorsinA knockout mice, blebbing is observed strictly in neural tissue (Goodchild et al., 2005), where TorsinA is highly expressed (Jungwirth et al., 2010). However, in fibroblasts from TorsinA knockout mice, additionally depleting TorsinB is sufficient to induce blebbing (Kim et al., 2010). In Torsin A knockout mice, blebbing is restricted to a specific developmental window, and the 


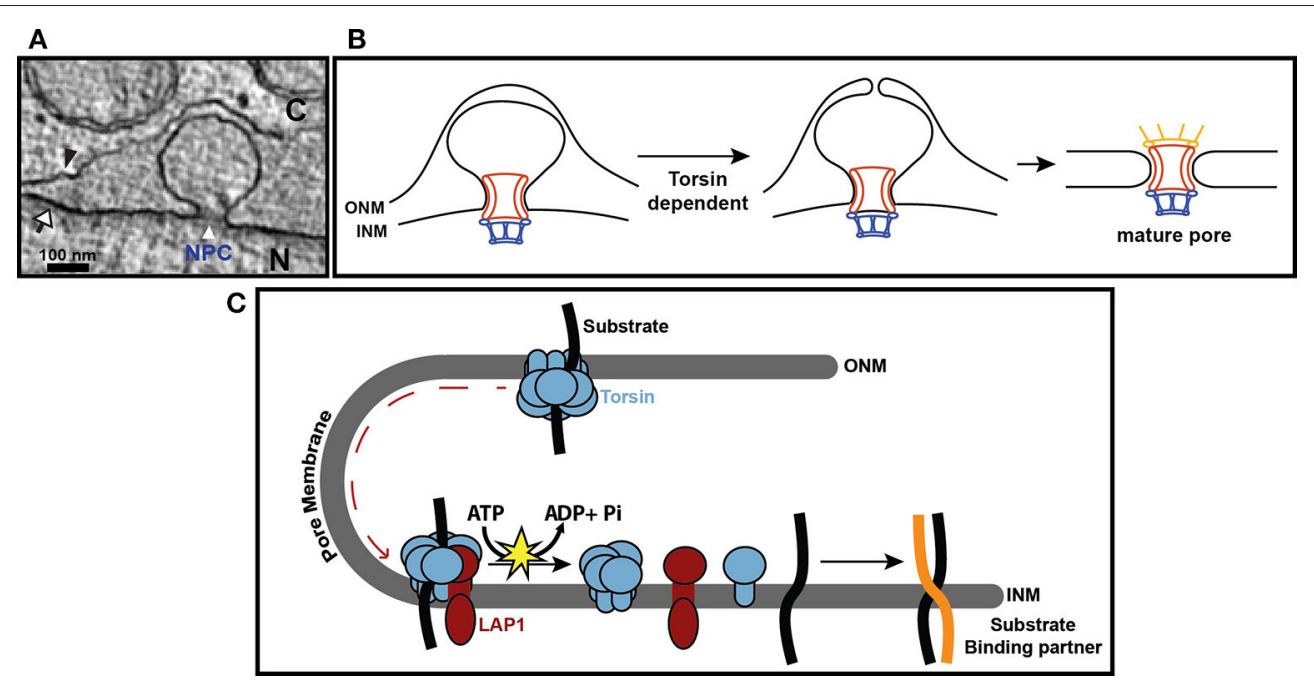

FIGURE 2 | Torsin function at the nuclear envelope. (A) EM cross section of nuclear envelope blebbing observed in Torsin-deficient HeLa cells. N, nucleus; C, cytoplasm; black arrowhead, ONM; white arrow, INM; white arrowhead, electron density at the base of the blebs containing nucleoporins. (B) Model depicting how blebs could arise from stalled NPC assembly. In this model, Torsin would function at a step prior to or at membrane fusion. (C) Hypothetical model for Torsins as a trafficking chaperones that deliver proteins to the inner nuclear membrane. INM-resident proteins are sequestered by Torsins during de novo synthesis in the ER or the contiguous ONM, preventing their premature assembly into protein-protein complexes that would compromise or prevent their trafficking through the pore membrane. Upon arrival at the INM, the high local concentration of LAP1 would trigger ATP hydrolysis in Torsins, leading to the disassembly of the Torsin ring and substrate release. Released substrates can then engage in protein-protein complex formation at the INM.

resolution of the blebs in later stages is dependent on increasing expression levels of TorsinB (Tanabe et al., 2016). Finally, deletion of TorsinA or TorsinB individually in HeLa cells shows little perturbation to normal nuclear envelope architecture, but deleting all four Torsins results in robust blebbing (Laudermilch et al., 2016).

While the precise composition of the blebs and Torsins' role in their formation is still being determined, several recent findings linked Torsins to nucleoporins (nups) (VanGompel et al., 2015; Laudermilch et al., 2016). In C. elegans, Torsin manipulation resulted in nup mislocalization and altered nuclear import kinetics (VanGompel et al., 2015). In Torsin-deficient HeLa cells, a subset of nups localize specifically to the base or "neck" of the blebs at the inner nuclear membrane (Laudermilch et al., 2016) (Figure 2A). Collectively, these observations suggest that Torsin plays a role in nuclear pore complex (NPC) biogenesis or homeostasis. The NPC is a massive structure found in the nuclear envelope through which nucleocytoplasmic transport occurs (Field et al., 2014; Knockenhauer and Schwartz, 2016; Kosinski et al., 2016; Lin et al., 2016). While the precise mechanism of NPC assembly is still actively investigated, there are two distinct assembly pathways: one occurs post-mitotically while the nuclear envelope reforms and the other occurs during interphase (Doucet et al., 2010). Interphase assembly begins from the INM and proceeds outward toward the ONM. After several subcomplexes have assembled, the inner and outer nuclear membranes fuse together, and at least some components of the cytoplasmic region are added to the NPC after this fusion event (Otsuka et al., 2016).

Here we propose two models for a functional link between Torsins and nups. Importantly, the shape and dimensions of the blebs are highly similar to normal interphase NPC assembly intermediates (Laudermilch et al., 2016; Otsuka et al., 2016). Thus, the blebs could represent frozen NPC assembly intermediates that require the action of Torsins for their completion. These intermediates would be frozen at a step prior to the fusion of the inner and outer nuclear membranes (Figure 2B). Thus, cytoplasmic nups would be expected to be absent from the base of the blebs in this model, while other subcomplexes would be present. Therefore, it will be critical to perform a detailed compositional analysis of the blebs. A diagnostic absence of cytoplasmic nups would support the idea of a frozen assembly intermediate. That Torsin-deficient cells remain viable albeit exhibiting slower growth (Laudermilch et al., 2016) could be attributed to the contribution of unperturbed NPC assembly proceeding through the Torsin-independent postmitotic insertion pathway.

Alternatively, the blebs could result from sealing of nascent NPCs by endosomal sorting complexes required for transport (ESCRT) components, analogous to a process that has recently been described in yeast in which ESCRT proteins and the $\mathrm{AAA}+$ ATPase Vps4 participate in a pathway that surveils NPCs (Webster et al., 2014; Webster and Lusk, 2016).

We envision two general mechanistic models to explain why blebs form in the absence of Torsin. In the first model, Torsin would act directly in NPC biogenesis. For example, Torsin might participate in the fusion of the inner and outer nuclear membranes during NPC assembly, probably in complex with other proteins. In the second model, Torsin would act upstream of NPC biogenesis or surveillance. Specifically, Torsins could act as trafficking chaperones by binding newly synthesized 
proteins in the endoplasmic reticulum and delivering them to sites of NPC assembly in the nuclear envelope (Figure 2C). Torsin could traffic transmembrane nups, or it could deliver proteins that are essential for NPC assembly or surveillance. One reason for invoking such a function is the presence of a 60 $\mathrm{kDa}$ transport limit for the nuclear domains of transmembrane proteins residing in the INM (Ungricht et al., 2015). NE proteins assembling into higher-order oligomeric structures must be held competent for trafficking through the pore membrane in a monomeric state to bypass the $60 \mathrm{kDa}$ size limitation imposed by the NPC. For example, trimeric Sun proteins (Sosa et al., 2012) at INM harbor sizable nuclear domains ( $\sim 34 \mathrm{kDa}$ for Sun1). Trafficking through the pore membrane in a trimeric state would be difficult to reconcile with this $60 \mathrm{kDa}$ size limit. Our specific proposal here is that Torsins could stabilize the monomeric form by association with the luminal domains of NE proteins, while the nuclear domains of NE proteins will ensure INM targeting. Upon arrival at the INM, substrates will be released from Torsins due to the high local concentration of the Torsin activator LAP1 at the INM resulting in disassembly of the Torsin ring and allowing the released substrate to engage in complex formation (Figure 2C). While hypothetical, this model would be consistent with the observation that a hydrolysis-deficient trap variant of TorsinA accumulates in the NE (Goodchild and Dauer, 2004; Naismith et al., 2004), which can be attributed to a failure of LAP1 to catalyze the release of Torsin from its NE-targeted clients.

Our model could also explain the accumulation of K48ubiquitylated proteins in the nuclear periphery in Torsin deficient cells (Laudermilch et al., 2016). Given that the INM of mammalian cells was recently shown to be competent for

\section{REFERENCES}

Blok, N. B., Tan, D., Wang, R. Y., Penczek, P. A., Baker, D., DiMaio, F., et al. (2015). Unique double-ring structure of the peroxisomal Pex1/Pex6 ATPase complex revealed by cryo-electron microscopy. Proc. Natl. Acad. Sci. U.S.A. 112, E4017-E4025. doi: 10.1073/pnas.1500257112

Brown, R. S., Zhao, C., Chase, A. R., Wang, J., and Schlieker, C. (2014). The mechanism of Torsin ATPase activation. Proc. Natl. Acad. Sci. U.S.A. 111, E4822-E4831. doi: 10.1073/pnas.1415271111

Cascalho, A., Jacquemyn, J., and Goodchild, R. E. (2017). Membrane defects and genetic redundancy: are we at a turning point for DYT1 dystonia? Mov. Disord. 32, 371-381. doi: 10.1002/mds. 26880

Chen, P., Burdette, A. J., Porter, J. C., Ricketts, J. C., Fox, S. A., Nery, F. C., et al. (2010). The early-onset torsion dystonia-associated protein, torsinA, is a homeostatic regulator of endoplasmic reticulum stress response. Hum. Mol. Genet. 19, 3502-3515. doi: 10.1093/hmg/ddq266

Demircioglu, F. E., Sosa, B. A., Ingram, J., Ploegh, H. L., and Schwartz, T. U. (2016). Structures of TorsinA and its disease-mutant complexed with an activator reveal the molecular basis for primary dystonia. Elife 5:17983. doi: 10.7554/eLife.17983

Doucet, C. M., Talamas, J. A., and Hetzer, M. W. (2010). Cell cycle-dependent differences in nuclear pore complex assembly in metazoa. Cell 141, 1030-1041. doi: 10.1016/j.cell.2010.04.036

Field, M. C., Koreny, L., and Rout, M. P. (2014). Enriching the pore: splendid complexity from humble origins. Traffic 15, 141-156. doi: 10.1111/tra.12141

Foisner, R., and Gerace, L. (1993). Integral membrane proteins of the nuclear envelope interact with lamins and chromosomes, and binding is modulated by mitotic phosphorylation. Cell 73, 1267-1279. doi: 10.1016/0092-8674(93)90355-T the degradation of membrane proteins (Tsai et al., 2016), it will be critical to determine if the half life of otherwise stable NPC/INM proteins (Doucet et al., 2010; Toyama et al., 2013) is compromised in Torsin-deficient cells due to the absence of normally stabilizing interactions that are perturbed due to trafficking defects, and to discern a (mis)localization of INM proteins to the ONM vs. INM upon Torsin manipulation.

In conclusion, we have now reached a stage in our understanding of Torsin biology that is sufficient to begin formulating more precise hypotheses about their mechanism and their functions that can be tested by definitive experiments. The likelihood that further genetic experiments within a cellular context will yield the holy grail of the Torsin fieldthe elusive substrates that trigger the changes affected by Torsins in the ER and at the nuclear envelope-is more probable than ever. Merging these functional details with a structural understanding of the Torsins' action will provide the necessary basis for developing targeted DYT1 dystonia therapies.

\section{AUTHOR CONTRIBUTIONS}

All authors listed, have made substantial, direct, and intellectual contribution to the work, and approved it for publication.

\section{ACKNOWLEDGMENTS}

This work was supported by the National Institutes of Health (1R01GM114401 to CS and T32GM007223 to EL) and an NSF GROW award to ARC.

Goodchild, R. E., Buchwalter, A. L., Naismith, T. V., Holbrook, K., Billion, K., Dauer, W. T., et al. (2015). Access of torsinA to the inner nuclear membrane is activity dependent and regulated in the endoplasmic reticulum. J. Cell Sci. 128, 2854-2865. doi: 10.1242/jcs. 167452

Goodchild, R. E., and Dauer, W. T. (2004). Mislocalization to the nuclear envelope: an effect of the dystonia-causing torsinA mutation. Proc. Natl. Acad. Sci. U.S.A. 101, 847-852. doi: 10.1073/pnas.0304375101

Goodchild, R. E., and Dauer, W. T. (2005). The AAA+ protein torsinA interacts with a conserved domain present in LAP1 and a novel ER protein. J. Cell Biol. 168, 855-862. doi: 10.1083/jcb.200411026

Goodchild, R. E., Kim, C. E., and Dauer, W. T. (2005). Loss of the dystoniaassociated protein torsinA selectively disrupts the neuronal nuclear envelope. Neuron 48, 923-932. doi: 10.1016/j.neuron.2005.11.010

Grillet, M., Dominguez Gonzalez, B., Sicart, A., Pottler, M., Cascalho, A., Billion, K., et al. (2016). Torsins are essential regulators of cellular lipid metabolism. Dev. Cell 38, 235-247. doi: 10.1016/j.devcel.2016.06.017

Hanson, P. I., and Whiteheart, S. W. (2005). AAA+ proteins: have engine, will work. Nat. Rev. Mol. Cell Biol. 6, 519-529. doi: 10.1038/nrm1684

Hedglin, M., Kumar, R., and Benkovic, S. J. (2013). Replication clamps and clamp loaders. Cold Spring Harb. Perspect. Biol. 5:a010165. doi: 10.1101/cshperspect.a010165

Jokhi, V., Ashley, J., Nunnari, J., Noma, A., Ito, N., Wakabayashi-Ito, N., et al. (2013). Torsin mediates primary envelopment of large ribonucleoprotein granules at the nuclear envelope. Cell Rep. 3, 988-995. doi: 10.1016/j.celrep.2013.03.015

Jungwirth, M., Dear, M. L., Brown, P., Holbrook, K., and Goodchild, R. (2010). Relative tissue expression of homologous torsinB correlates with the neuronal specific importance of DYT1 dystonia-associated torsinA. Hum. Mol. Genet. 19, 888-900. doi: 10.1093/hmg/ddp557 
Kelch, B. A. (2016). Review: the lord of the rings: structure and mechanism of the sliding clamp loader. Biopolymers 105, 532-546. doi: 10.1002/bip.22827

Kim, C. E., Perez, A., Perkins, G., Ellisman, M. H., and Dauer, W. T. (2010). A molecular mechanism underlying the neural-specific defect in torsinA mutant mice. Proc. Natl. Acad. Sci. U.S.A. 107, 9861-9866. doi: 10.1073/pnas.0912877107

Knockenhauer, K. E., and Schwartz, T. U. (2016). The nuclear pore complex as a flexible and dynamic gate. Cell 164, 1162-1171. doi: 10.1016/j.cell.2016.01.034

Kosinski, J., Mosalaganti, S., von Appen, A., Teimer, R., DiGuilio, A. L., Wan, W., et al. (2016). Molecular architecture of the inner ring scaffold of the human nuclear pore complex. Science 352, 363-365. doi: 10.1126/science.aaf0643

Laudermilch, E., and Schlieker, C. (2016). Torsin ATPases: structural insights and functional perspectives. Curr. Opin. Cell Biol. 40, 1-7. doi: 10.1016/j.ceb.2016.01.001

Laudermilch, E., Tsai, P. L., Graham, M., Turner, E., Zhao, C., and Schlieker, C. (2016). Dissecting Torsin/cofactor function at the nuclear envelope: a genetic study. Mol. Biol. Cell 27, 3964-3971. doi: 10.1091/mbc.E16-07-0511

Liang, C. C., Tanabe, L. M., Jou, S., Chi, F., and Dauer, W. T. (2014). TorsinA hypofunction causes abnormal twisting movements and sensorimotor circuit neurodegeneration. J. Clin. Invest. 124, 3080-3092. doi: 10.1172/JCI72830

Lin, D. H., Stuwe, T., Schilbach, S., Rundlet, E. J., Perriches, T., Mobbs, G., et al. (2016). Architecture of the symmetric core of the nuclear pore. Science 352:aaf1015. doi: 10.1126/science.aaf1015

Luxton, G. W., Gomes, E. R., Folker, E. S., Worman, H. J., and Gundersen, G. G. (2011). TAN lines: a novel nuclear envelope structure involved in nuclear positioning. Nucleus 2, 173-181. doi: 10.4161/nucl.2.3.16243

Martin, A., Baker, T. A., and Sauer, R. T. (2008). Pore loops of the AAA+ ClpX machine grip substrates to drive translocation and unfolding. Nat. Struct. Mol. Biol. 15, 1147-1151. doi: 10.1038/nsmb.1503

Mogk, A., Schlieker, C., Strub, C., Rist, W., Weibezahn, J., and Bukau, B. (2003). Roles of individual domains and conserved motifs of the AAA+ chaperone $\mathrm{ClpB}$ in oligomerization, ATP hydrolysis, and chaperone activity. J. Biol. Chem. 278, 17615-17624. doi: 10.1074/jbc.M209686200

Naismith, T. V., Dalal, S., and Hanson, P. I. (2009). Interaction of torsinA with its major binding partners is impaired by the dystonia-associated DeltaGAG deletion. J. Biol. Chem. 284, 27866-27874. doi: 10.1074/jbc.M109.020164

Naismith, T. V., Heuser, J. E., Breakefield, X. O., and Hanson, P. I. (2004). TorsinA in the nuclear envelope. Proc. Natl. Acad. Sci. U.S.A. 101, 7612-7617. doi: 10.1073/pnas.0308760101

Nery, F. C., Armata, I. A., Farley, J. E., Cho, J. A., Yaqub, U., Chen, P., et al. (2011). TorsinA participates in endoplasmic reticulum-associated degradation. Nat. Commun. 2:393. doi: 10.1038/ncomms1383

Olivares, A. O., Baker, T. A., and Sauer, R. T. (2016). Mechanistic insights into bacterial AAA+ proteases and protein-remodelling machines. Nat. Rev. Microbiol. 14, 33-44. doi: 10.1038/nrmicro.2015.4

Otsuka, S., Bui, K. H., Schorb, M., Hossain, M. J., Politi, A. Z., Koch, B., et al. (2016). Nuclear pore assembly proceeds by an inside-out extrusion of the nuclear envelope. Elife 5:e19071. doi: 10.7554/eLife.19071.

Ozelius, L. J., Hewett, J. W., Page, C. E., Bressman, S. B., Kramer, P. L., Shalish, C., et al. (1997). The early-onset torsion dystonia gene (DYT1) encodes an ATP-binding protein. Nat. Genet. 17, 40-48. doi: 10.1038/ng0997-40

Pappas, S. S., Darr, K., Holley, S. M., Cepeda, C., Mabrouk, O. S., Wong, J. M., et al. (2015). Forebrain deletion of the dystonia protein torsinA causes dystoniclike movements and loss of striatal cholinergic neurons. Elife 4:e08352. doi: $10.7554 /$ elife. 08352

Rose, A. E., Brown, R. S., and Schlieker, C. (2015). Torsins: not your typical AAA+ ATPases. Crit. Rev. Biochem. Mol. Biol. 50, 532-549. doi: 10.3109/10409238.2015.1091804

Rose, A. E., Zhao, C., Turner, E. M., Steyer, A. M., and Schlieker, C. (2014). Arresting a Torsin ATPase reshapes the endoplasmic reticulum. J. Biol. Chem. 289, 552-564. doi: 10.1074/jbc.M113.515791

Saunders, C. A., Harris, N. J., Willey, P. T., Woolums, B. M., Wang, Y., McQuown, A. J., et al. (2017). TorsinA controls TAN line assembly and the retrograde flow of dorsal perinuclear actin cables during rearward nuclear movement. J. Cell Biol. 216, 657-674. doi: 10.1083/jcb.201507113

Scheffzek, K., Ahmadian, M. R., and Wittinghofer, A. (1998). GTPaseactivating proteins: helping hands to complement an active site.
Trends Biochem. Sci. 23, 257-262. doi: 10.1016/S0968-0004(98) 01224-9

Sosa, B. A., Demircioglu, F. E., Chen, J. Z., Ingram, J., Ploegh, H., and Schwartz, T. U. (2014). How lamina-associated polypeptide 1 (LAP1) activates Torsin. Elife 3:e03239. doi: 10.7554/elife.03239

Sosa, B. A., Rothballer, A., Kutay, U., and Schwartz, T. U. (2012). LINC Complexes Form by Binding of three Kash peptides to domain interfaces of trimeric Sun Proteins. Cell 149, 1035-1047. doi: 10.1016/j.cell.2012.03.046

Tanabe, L. M., Liang, C. C., and Dauer, W. T. (2016). Neuronal nuclear membrane budding occurs during a developmental window modulated by torsin paralogs. Cell Rep. 16, 3322-3333. doi: 10.1016/j.celrep.2016.08.044

Toyama, B. H., Savas, J. N., Park, S. K., Harris, M. S., Ingolia, N. T., Yates, J. R. III. et al. (2013). Identification of long-lived proteins reveals exceptional stability of essential cellular structures. Cell 154, 971-982. doi: 10.1016/j.cell.2013. 07.037

Tsai, P. L., Zhao, C., Turner, E., and Schlieker, C. (2016). The Lamin B receptor is essential for cholesterol synthesis and perturbed by disease-causing mutations. Elife 5:e16011. doi: 10.7554/eLife.16011.

Ungricht, R., Klann, M., Horvath, P., and Kutay, U. (2015). Diffusion and retention are major determinants of protein targeting to the inner nuclear membrane. J. Cell Biol. 209, 687-703. doi: 10.1083/jcb.201409127

Vander Heyden, A. B., Naismith, T. V., Snapp, E. L., Hodzic, D., and Hanson, P. I. (2009). LULL1 retargets TorsinA to the nuclear envelope revealing an activity that is impaired by the DYT1 dystonia mutation. Mol. Biol. Cell 20, 2661-2672. doi: 10.1091/mbc.E09-01-0094

VanGompel, M. J. W., Nguyen, K. C. Q., Hall, D. H., Dauer, W. T., and Rose, L. S. (2015). A novel function for the Caenorhabditis elegans torsin OOC-5 in nucleoporin localization and nuclear import. Mol. Biol. Cell 26, 1752-1763. doi: 10.1091/mbc.E14-07-1239

Webster, B., M., Colombi, P., Jager, J., and Lusk, C. P. (2014). Surveillance of nuclear pore complex assembly by ESCRT-III/Vps4. Cell 159, 388-401. doi: 10.1016/j.cell.2014.09.012

Webster, B. M., and Lusk, C. P. (2016). Border safety: quality control at the nuclear envelope. Trends Cell Biol. 26, 29-39. doi: 10.1016/j.tcb.2015.08.002

Yokom, A. L., Gates, S. N., Jackrel, M. E., Mack, K. L., Su, M., Shorter, J., et al. (2016). Spiral architecture of the Hsp104 disaggregase reveals the basis for polypeptide translocation. Nat. Struct. Mol. Biol. 23, 830-837. doi: 10.1038/nsmb. 3277

Zhao, C., Brown, R. S., Chase, A. R., Eisele, M. R., and Schlieker, C. (2013). Regulation of Torsin ATPases by LAP1 and LULL1. Proc. Natl. Acad. Sci. U.S.A. 110, E1545-E1554. doi: 10.1073/pnas.1300676110

Zhao, C., Brown, R. S., Tang, C. H., Hu, C. C., and Schlieker, C. (2016). Sitespecific proteolysis mobilizes torsina from the membrane of the Endoplasmic Reticulum (ER) in response to ER Stress and B cell stimulation. J. Biol. Chem. 291, 9469-9481. doi: 10.1074/jbc.M115.709337

Zhao, M., Wu, S., Zhou, Q., Vivona, S., Cipriano, D. J., Cheng, Y., et al. (2015). Mechanistic insights into the recycling machine of the SNARE complex. Nature 518, 61-67. doi: 10.1038/nature14148

Zhu, L., Millen, L., Mendoza, J. L., and Thomas, P. J. (2010). A unique redoxsensing sensor II motif in TorsinA plays a critical role in nucleotide and partner binding. J. Biol. Chem. 285, 37271-37280. doi: 10.1074/jbc.M110. 123471

Zhu, L., Wrabl, J. O., Hayashi, A. P., Rose, L. S., and Thomas, P. J. (2008). The torsin-family AAA+ protein OOC- 5 contains a critical disulfide adjacent to Sensor-II that couples redox state to nucleotide binding. Mol. Biol. Cell 19, 3599-3612. doi: 10.1091/mbc.E08-01-0015

Conflict of Interest Statement: The authors declare that the research was conducted in the absence of any commercial or financial relationships that could be construed as a potential conflict of interest.

Copyright (c) 2017 Chase, Laudermilch and Schlieker. This is an open-access article distributed under the terms of the Creative Commons Attribution License (CC BY). The use, distribution or reproduction in other forums is permitted, provided the original author(s) or licensor are credited and that the original publication in this journal is cited, in accordance with accepted academic practice. No use, distribution or reproduction is permitted which does not comply with these terms. 